\title{
Major Histocompatibility Complex Tested Indicator
}

National Cancer Institute

\section{Source}

National Cancer Institute. Major Histocompatibility Complex Tested Indicator. NCI

Thesaurus. Code C158347.

An indication as to whether the study subject has had its major histocompatibility complex characterized. 